\title{
Accuracy of Reporting Primary Care Specialty Status in Medical Research
}

\author{
Andrea M. Diep, Harish S. Thoppe, BS, Angela Yang, Abhinav S. Agnani, and \\ William R. Phillips, MD, MPH
}

Introduction: Family physicians (FPs) are specialty trained and certified and provide most primary care (PC) services in the United States. General practitioners (GPs), a separate group without specialty PC training, are commonly confused with FPs despite differences in demographic characteristics, professional qualifications, and clinical services. Our study documents how often research in major medical journals distinguishes between these 2 groups or combines GPs and FPs together.

Methods: We selected 23 US journals on impact factor and relevance to PC. Using a MEDLINE search, we identified all reports published in 2016 through 2017 that met inclusion criteria: original research; done in United States; studying FPs, GPs, and/or PC physicians. Two researchers reviewed each article to determine inclusion and whether it lumped or split FPs and GPs.

Results: Search retrieved 409 total studies, with 88 (21.5\%) meeting inclusion criteria. Among these, $35(39.8 \%)$ included FPs only, leaving 53 (60.2\%) that also included GPs. Among these studies, only 3 (5.7\%) separated GPs from FPs. Another 21 (39.6\%) combined GPs and FPs together. In 29 (54.7\%), the classification of GPs, FPs and others was not described.

Conclusions: Most PC research reports combine GPs and FPs into a single group, masking differences between these distinct groups. Most research reports fail to explain how they classify PC clinicians. Research reports need to improve classification of FPs and PC clinicians. (J Am Board Fam Med 2019;32:941-943.)

Keywords: Family Physicians, General Practitioners, Internship and Residency, Journal Impact Factor, MEDLINE, Primary Health Care, Research Report, Specialization

Primary care (PC) is the foundation of health care in the United States. The family physician (FP) is the physician specialty trained in comprehensive PC for all patients. Historically, general practitioners (GPs) practiced general medicine, usually without specific training in PC. ${ }^{1}$ Family medicine was recognized as a distinct specialty by the American Board of Medical Specialties in 1969, with prescribed residency training and cer-

This article was externally peer reviewed.

Submitted 15 April 2019; revised 1 July 2019; accepted 3 July 2019.

From the University of Washington, Seattle, WA (AMD, HST, AY, ASA); Department of Family Medicine, University of Washington, Seattle, WA (WRP)

Funding: none.

Conflict of interest: none declared.

Corresponding author: William R. Phillips, MD, MPH, Department of Family Medicine, University of Washington, Box 356390, Seattle, WA, 98195-6390, (E-mail: wphllps@uw.edu). tification by the American Board of Family Medicine. $^{2}$ A substantial group of physicians currently in practice and entering the workforce still call themselves GPs, but differ markedly from FPs in demographic characteristics, professional qualifications, and clinical services. ${ }^{3}$ Physicians who self-identify as GPs comprise approximately $10 \%$ (6661) of all FPs-plus-GPs in the United States, a group that outnumbers 25 of 44 specialties recognized by American Board of Medical Specialties (ABMS), including medicine/pediatrics, geriatrics, and preventive medicine. ${ }^{4}$ Thus, GPs constitute a significant component of US physician workforce and particularly of the PC workforce. Compared with FPs, GPs are more likely to be older, male, DOs, graduates of non-US medical schools; to have no residency training in family medicine or in any field related to primary care; and are less likely to participate in Medicare or work in hospitals. ${ }^{3}$ 
Figure 1. Research articles on FPs and GPs published in 23 medical journals 2016-17. FP, family physicians; GP, general practitioners; PC, primary care.

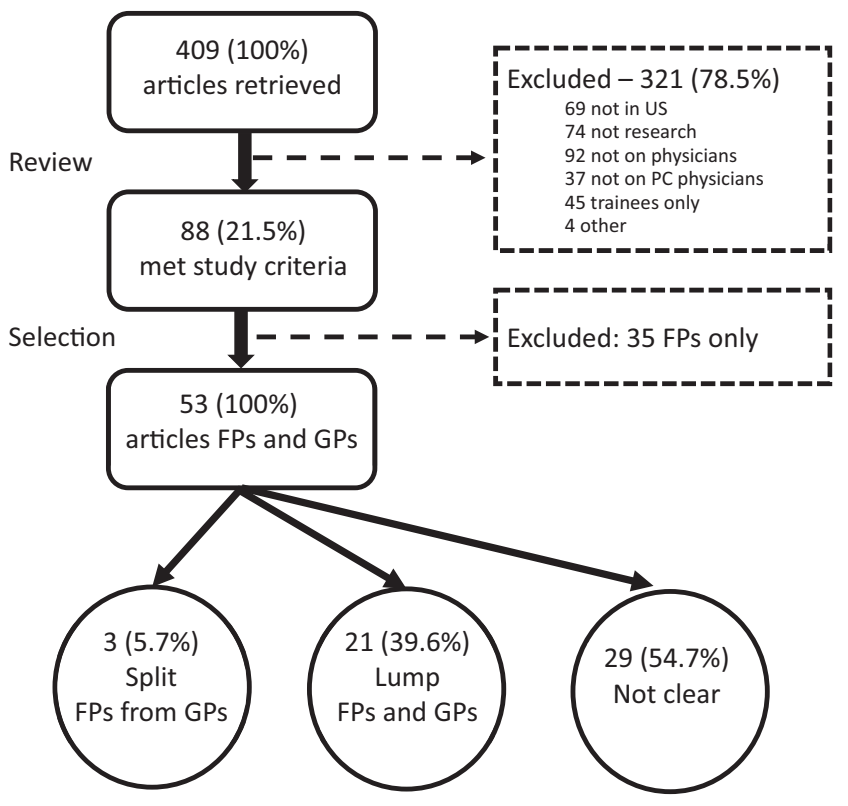

Many medical and health care researchers, however, combine these 2 groups together in an aggregate FP/GP group, risking misclassification bias and potentially obscuring important differences between groups. Our study aimed to document the rate at which research in major medical journals splits or lumps GPs and FPs.

\section{Methods}

We selected 23 major US medical journals for relevance to PC research and impact factor, a metric of the journal's yearly average number of citations to recent articles published in a that journal. ${ }^{5}$ Systematic MEDLINE search identified all articles meeting study criteria: published in 2016 through 2017; original research; studying FPs, GPs, and/or PC physicians in the United States. Two researchers reviewed each article to determine inclusion and whether it reported FPs and GPs as separate groups or combined them into an aggregate group. (See Appendix for details of study journals and MEDLINE search string.)

\section{Results}

See Figure 1 for details on articles and reporting of FP and GP groups. Across the 23 journals, the literature search retrieved 409 studies, with 88 (21.5\%) meeting inclusion criteria. Among these, 35 (39.8\%) included FPs only, leaving 53 (60.2\%) that included GPs and FPs. Among studies including GPs, only 3 (5.7\%) separated GPs from FPs. Another 21 (39.6\%) combined the GPs and FPs into 1 group. In 29 (54.7\%), the reporting of GPs, FPs, and others was not clearly described. We found no studies on GPs only and none that combined GPs with internal medicine or pediatrics physicians.

\section{Conclusions}

Our study documents that research on PC usually fails to identify physicians by specialty. This failure represents a misunderstanding of modern PC physicians, practices and systems.

Most research (55\%) reports a mix FPs, GPs and other PC clinicians and fails to adequately describe their categories or methods. Most research focusing on FPs and GPs (40\%) combines them together into an aggregate group. Few reports (5.7\%) accurately split FPs and GPs into separate groups. Mixing these distinct groups can bias results and obscure important differences between clinician groups. Errors in classifying up to $10 \%$ of FP physicians may affect research results and conclusions, particularly when testing for differences between groups of physicians and patient outcomes related to physician age, sex, medical school, postgraduate training, PC training, and practice patterns. 
This is the first study to quantify these inappropriate research and reporting practices. Study strengths include our formal methods for literature search and review of a large number of current research reports. A limitation might be the selection of journals. Studies involving PC appear in many journals and specialty areas and a broader set of journals might yield different results. However, we selected journals most likely to focus on PC research and it seems that other journals, from more distant fields, would be even more likely to make these reporting errors. In addition, we did not focus on research on other PC clinicians: general internists, general pediatricians, nurse practitioners, and physician assistants. Further analysis of FP-GP reporting practices by journal type, author specialty, and source of specialty information was limited by small numbers in these subgroups.

Based on these data, we recommend that researchers consider FPs, GPs, and other PC clinicians separately in planning, conducting and reporting research. Journal editors, reviewers, and readers should insist that research reports do not combine FPs and GPs together.

Acknowledgements: We thank Sarah Safranek, MLIS, University of Washington Health Sciences Library, for her assistance with literature search methods.

To see this article online, please go to: http://jabfm.org/content/ 32/6/941.full.

\section{References}

1. Ad Hoc Committee on Education for Family Practice (Willard Committee). Meeting the challenge of family practice. Chicago, IL: American Medical Association; 1966.

2. Adams D. American Board of Family Practice. A bistory. Lexington, KY: American Board of Family Practice; 1999.

3. Phillips WR, Dai M, Frey J, Peterson LE. General practitioners in US medical practice compared to family physicians. Ann Fam Med 2019 (In Press).

4. Association of American Medical Colleges. Washington, D.C. 2018 Physician Specialty Data Report. Available from: https://www.aamc.org/data/workforce/reports/492558/1-2-chart.html. Accessed February $28,2019$.

5. Clarivate Analytics. InCites Journal Citation Reports. 2017. Available from: https://jcr-clarivate-com. 


\section{APPENDIX}

\section{PubMed MEDLINE Literature Search String}

Example below for New England Fournal of Medicine, 2017.

(general practitioners[mh] OR "general practitioner"[tiab] OR "general practitioners"[tiab] OR family physicians[mh] OR "family physician"[tiab] OR "family physicians" [tiab] OR primary care physicians [mh] primary care physician*[tiab] OR primary health care physician*[tiab] OR primary health care physician*[tiab] OR ((general practice[mh] OR family practice[mh] OR primary health care[mh] OR "general medicine"[tiab] OR general practice*[tiab] OR generalist*[tiab] OR family practice* [tiab] OR family medicine[tiab] OR primary care[tiab] OR "primary health care"[tiab] OR "primary health care"[tiab] OR "primary medical care"[tiab]) AND (doctor[tiab] OR doctors[tiab] OR physician[tiab] OR physician$s$ [tiab] OR practitioner[tiab] OR practitioners[tiab] OR physicians[mh])))
AND 2017[pdat] AND english[la]

NOT

(addresses[pt] OR bibliography[pt] OR case reports[pt] OR clinical conference[pt] OR congresses[pt] OR consensus development conference[pt] OR dataset[pt] OR dictionary[pt] OR directory[pt] OR duplicate publication[pt] OR editorial[pt] OR expression of concern[pt] OR government publications[pt] OR guideline[pt] OR historic article[pt] OR interactive tutorial[pt] $\mathrm{OR}$ introductory journal article[pt] OR lectures[pt] OR legal cases[pt] OR legislation[pt] OR news[pt] OR newspaper article[pt] OR patient education handout[pt] OR periodical index[pt] OR portraits[pt] OR practice guideline[pt] OR scientific integrity review[pt] OR support of research[pt] OR twin study[pt])

AND

$n$ engl j med[ta] 
Appendix: Medical Journals Reviewed for Studies Reporting on FPs and GPs, 2016 to 2017

\begin{tabular}{|c|c|c|c|c|c|}
\hline Journal Title & $\begin{array}{l}\text { InCites } \\
\text { Category* }^{*}\end{array}$ & $\begin{array}{l}\text { Category } \\
\text { Rank}^{*}\end{array}$ & $\begin{array}{l}\text { Impact } \\
\text { Factor* }\end{array}$ & $\begin{array}{l}\text { Articles } \\
\text { Retrieved }\end{array}$ & $\begin{array}{l}\text { Articles } \\
\text { Included }\end{array}$ \\
\hline Total & - & - & - & $409(100 \%)$ & $\begin{array}{c}88 \\
(100 \%)\end{array}$ \\
\hline Annals of Family Medicine & PHC & 1 & 4.540 & 20 & 4 \\
\hline 7 American Board of Family Med & $\mathrm{PHC}$ & 3 & 2.515 & 67 & 17 \\
\hline Family Practice & PHC & 11 & 1.675 & 25 & 1 \\
\hline Family Medicine & PHC & 15 & 1.140 & 86 & 10 \\
\hline Fournal of Family Practice & $\mathrm{PHC}$ & 19 & 0.534 & 0 & 0 \\
\hline New England Fournal of Medicine & MGI & 1 & 79.258 & 4 & 1 \\
\hline $\begin{array}{l}\text { fAMA-7 American Medical } \\
\text { Assoc }\end{array}$ & MGI & 3 & 47.661 & 4 & 1 \\
\hline fAMA Internal Medicine & MGI & 5 & 19.989 & 15 & 4 \\
\hline fAMA Pediatrics & PED & 1 & 10.769 & 4 & 1 \\
\hline Pediatrics & PED & 3 & 5.515 & 17 & 2 \\
\hline Fournal of Pediatrics & PED & 8 & 3.667 & 15 & 4 \\
\hline Academic Pediatrics & PED & 22 & 2.806 & 15 & 4 \\
\hline American Fournal of Public Health & PHEALTH & 19 & 4.380 & 5 & 1 \\
\hline American 7 Preventive Medicine & PHEALTH & 30 & 4.127 & 12 & 5 \\
\hline Preventive Medicine & PHEALTH & 43 & 3.483 & 18 & 5 \\
\hline Medical Care & PHEALTH & 45 & 3.338 & 5 & 1 \\
\hline Health Affairs & HSERV & 4 & 4.843 & 0 & 0 \\
\hline Academic Medicine & HSERV & 5 & 4.801 & 24 & 3 \\
\hline 7 General Internal Medicine & HSERV & 13 & 4.005 & 54 & 13 \\
\hline Medical Care & HSERV & 16 & 3.338 & 5 & 1 \\
\hline Health Services Research & HSERV & 24 & 2.667 & 18 & 7 \\
\hline Fournal of Rural Health & HSERV & 59 & 1.762 & 6 & 2 \\
\hline Fournal of Interprofessional Care & HSERV & 61 & 1.601 & 0 & 0 \\
\hline Evaluation Health Professions & HSERV & 64 & 1.531 & 0 & 0 \\
\hline
\end{tabular}

FP, family physicians; GP, general practitioners.

Journals selected from InCItes* categories: PHC = Primary Health Care; MGI = Medicine, General \& Internal; PED = Pediatrics; PHEALTH $=$ Public, Environmental \& Occupational Health; HSERV $=$ Health Care Sciences \& Services. Some journals appear in more than one category.

*Clarivate Analytics. InCites Journal Citation Reports. Published 2017.

https://jcr-clarivate-com. Accessed March 22, 2019. 